QUARTERLY OF APPLIED MATHEMATICS

VOLUME LXIII, NUMBER 2

JUNE 2005, PAGES 309-324

$\mathrm{S} 0033-569 \mathrm{X}(05) 00950-0$

Article electronically published on February 28, 2005

\title{
A HAMILTON-JACOBI APPROACH TO THE CONTROL OF THE TRAPPING TIME OF A SOLITON BY AN EXTERNAL POTENTIAL
}

\author{
BY \\ G. KOSSIORIS (Department of Mathematics, University of Crete, 71409 Heraklion, Greece AND \\ Institute of Applied and Computational Mathematics, FORTH, 71110 Heraklion, Greece), \\ M. PLEXOUSAKIS (Department of Applied Mathematics, University of Crete, 71409 Heraklion, \\ Greece AND Institute of Applied and Computational Mathematics, FORTH, 71110 Heraklion, Greece), \\ AND \\ A. N. YANNACOPOUlOS (Department of Statistics and Actuarial Science, University of the \\ Aegean, 82300 Karlovassi, Samos, Greece) \\ Abstract. The control of the trapping time of a localized solution of the nonlinear \\ Schrödinger equation (NLS) with the use of an external parabolic potential is studied. \\ We reduce the dynamics of the position of the soliton center to those of a controlled \\ linear oscillator and then study the viscosity solution of the associated Hamilton-Jacobi \\ equation. A numerical scheme is proposed for the treatment of the problem.
}

1. Introduction. Soliton control is an important problem in applied mathematics. There is a wide range of applications where solitary waves appear, e.g., biological systems, optical systems, fluid mechanics, etc., and in most cases it is useful to provide control mechanisms that can drive the soliton to a desired state within a given time interval.

The particular model we wish to study in this paper is a nonlinear Schrödinger equation (NLS) with external potential and dissipation in the form

$$
i\left(u_{t}+\gamma u+C \nabla u\right)-\frac{1}{2} D \Delta u+a f\left(|u|^{2}\right) u+\sigma U(x) u=0,
$$

where $u$ is a complex-valued envelope of a wave field, $x \in \mathbb{R}^{n}$, and $f$ is a real-valued function. The coefficient $\gamma$ is a coefficient modeling dissipation in the medium and $D$ is the dispersion coefficient of the medium. $U(x, t)$ is an external potential which models the inhomogeneity properties of the medium, and which will be allowed to be random here. This model is a well-established model in a wide range of applications, such as

Received June, 2004.

2000 Mathematics Subject Classification. Primary 49L25.

E-mail address: kosioris@math.uoc.gr

E-mail address: plex@tem.uoc.gr

E-mail address: ayannaco@aegean.gr

(C)2005 Brown University 
fiber optics or fluid mechanics. For instance, in the context of nonlinear optics, $u$ describes the complex envelope of an electromagnetic field $\mathbf{E}(z, t)=\operatorname{Re}\left[U(z, t) e^{i\left(k_{0} z-\omega_{0} t\right)}\right] \hat{\mathbf{y}}$ propagating in an optical fiber with index of refraction $k=\frac{\omega}{c} n_{0}(\omega)+\frac{\omega}{c} n_{2}|\mathbf{E}|^{2}$. The potential term $U$ can be shown to arise from inhomogeneities of the medium or even as an external potential [10. In the context of atomic physics, this model can serve as a model for Bose-Einstein condensation (BEC), where now $u$ is the wave function of the state of some atomic system and $U$ is an external potential (potential trap) needed for the confinement of the condensate [11].

The NLS equation is known to possess, under certain conditions, localized wave solutions called solitary waves (in the sequel we shall refer to them under the general name solitons, although in the higher dimensional case they may not be strictly solitons).

The external potential is expected to have some effect on the motion of the solitons. For instance, under the effect of the potential, the soliton may be set to motion or may undergo radiation emission, etc., [9. The question we wish to address here is the possibility of using the external potential in the control of the soliton, that is, whether the external potential can be chosen in such a way as to maximize or minimize the expected trapping time of the soliton in the potential. The problem will be addressed here using techniques from control theory and, in particular, the Hamilton-Jacobi equation approach. The full treatment of the problem would require the control of an infinite dimensional problem (the NLS partial differential equation). While the subject of control of infinite dimensional systems is a fascinating subject that has attracted the attention of many researchers in the field of applied mathematics, we choose here an alternate approach which is also of considerable interest. As we show in this paper, for the special case of parabolic spatial potentials, assuming that a soliton exists, its center of mass should satisfy a finite dimensional system (an ordinary differential equation) in the form of a linear oscillator with varying frequency. Therefore, the problem of controlling the soliton (steering it into a desired domain) with the use of the potential as a control can be translated into the problem of controlling a linear oscillator. It is the aim of the present paper to study this simplified control problem, with the use of viscosity solutions of the Hamilton-Jacobi equation associated with the ODE control problem. The full problem is the subject of current investigation.

The remainder of the paper is organized as follows: In Section 2 we formulate the problem and derive the ordinary differential equation satisfied by the center of mass of the soliton and we formulate the control problem. In Section 3 we derive the HamiltonJacobi equation associated with the control problem under investigation and present the connection of the viscosity solutions of this equation with the minimum time needed in order to steer the soliton into the desired target. In Section 4 we propose a numerical scheme for the calculation of the value function and the control protocol. Finally, in Section 5 we present our conclusions.

2. Formulation of the problem. The problem we wish to study is the following: Assume that the NLS model described by equation (1.1) admits a localized travelling wave solution $u(x, t)$, which will be called from now on a soliton. Can we choose the potential $U$ so as to steer the center of mass of the soliton into a given target $G$ in the 
minimum time? The first step in the study of this problem is to obtain an equation for the motion of the soliton center. We may define the position of the soliton center by the vector

$$
X=\int_{\mathbb{R}^{n}} x|u|^{2} d x
$$

Differentiating twice with respect to time and using the NLS equation (1.1) we find, after a few integrations by parts, that the position of the soliton center satisfies the ordinary differential equation

$$
\ddot{X}=-4 \gamma \dot{X}-4 \gamma^{2} X-2 \int_{\mathbb{R}^{n}} \nabla U|u|^{2} d x,
$$

which, upon the change of variables $X^{\prime} \rightarrow X \exp (-2 \gamma t)$, becomes

$$
\ddot{X}^{\prime}=-2 \exp (2 \gamma t) \int_{\mathbb{R}^{n}} \nabla U|u|^{2} d x .
$$

This equation was derived in [13] (see also 14]).

This equation is not in closed form and has to be treated together with the original NLS equation in order to provide results for the motion of the soliton center. In the special case where the potential is quadratic, the above equation may be closed and can be rendered in the form of a linear oscillator (see [13]).

In the general case, however, we may make some simplifying assumptions which turn equation (2.1) into a stochastic ODE. We assume that the potential is slowly varying as a function of space, i.e., $U(x, t)=U(\epsilon x, t)$. Under this assumption and since the soliton solution is localized in space, we may simplify equation (2.1) into an ODE that corresponds to the equation of motion of a particle in a general potential that is subject to stochastic perturbations (in time). The general form will be

$$
\ddot{X}=-\nabla U(X, t) .
$$

The second step consists of addressing the question of choosing the potential $U$ in such a way as to minimize the time in which the particle enters some specified area covered by the potential.

We shall assume that the external potential consists of a parabolic part plus some, unknown to this point, part which we will assume to be the control part of the potential

$$
U(x, t)=\sigma(t) x^{T} A x+u(t) u_{c}(\epsilon x) .
$$

Under this assumption the equation of motion for the soliton center becomes

$$
\ddot{X}=-\sigma(t) A X-u(t) \nabla u_{c}(X) .
$$

Equation (2.3) is an approximate equation for the case of general forms of $u_{c}$. The slow dependence of $u_{c}$ on space allows us to obtain an equation for the motion of the soliton center in closed form. However, in the particular case where $u_{c}$ is assumed to be parabolic, equation (2.3) is exact and becomes

$$
\ddot{X}=-\sigma(t) A X-u(t) B X .
$$


The problem we wish to study can now be formulated as follows: Suppose that the soliton starts outside a chosen region $G$. How can we choose $u(t)$ and $u_{c}$ in such a way as to minimize the entry time of the soliton in a region of the potential?

We will treat the above problem using the methods of control theory, and in particular through the use of the Hamilton-Jacobi equation. We need the following definitions:

Let $G$ be the target set into which we wish to drive the soliton. We consider $G$ to be a closed set with compact boundary $\partial G$. We will consider the initial state $x \in G^{c}:=\mathbb{R}^{N} \backslash G$. By $X_{x}(t, u)$ we denote the trajectory of the system that started at initial condition $x$, having adopted the control protocol $u$.

Definition 2.1. We define the entry time in the set $G, t_{x}(u)$ as follows:

$$
t_{x}(u)= \begin{cases}+\infty, & \text { if }\left\{t: X_{x}(t, u) \in G\right\}=\emptyset \\ \min \left\{t: X_{x}(t, u) \in G\right\}, & \text { otherwise }\end{cases}
$$

The minimal time function $T(x)$ is defined as

$$
T(x)=\inf _{u \in \mathcal{A}} t_{x}(u), \quad x \in \mathbb{R}^{N},
$$

where $\mathcal{A}$ is, in general, the set of measurable functions $u:[0, \infty) \rightarrow A$ with $A$ a compact subset of $\mathbb{R}^{M}$.

For the specific problem treated here, we have $N=2$ and $M=2$. The minimal time function is the minimal time needed for the trajectory that has started in $x$ to enter into the set $G$. The problem at hand is now a standard control problem. It may be reformulated as: Find the control protocol $u$ such that $T(x)$ is attained when $X(t)$ is subject to the dynamics

$$
\begin{aligned}
& \dot{X}(t)=b(t, X(t), u(t)), \\
& X(0)=x .
\end{aligned}
$$

We will restrict ourselves to the case where the control potential is parabolic so as to have an exact form of the equation for the soliton center. In this case, $b(t, X(t), u(t))$ is the vector field corresponding to a controlled linear oscillator described by equation (2.4).

3. The Hamilton-Jacobi approach to the control of a linear oscillator. In this section we present the formulation of the exit time control for the linear oscillator describing the motion of the soliton center through the use of viscosity solutions of Hamilton-Jacobi equations.

The dynamical system to be controlled is of the following form:

$$
\begin{aligned}
& \dot{x}_{1}=x_{2}, \\
& \dot{x}_{2}=-\left(1+u_{1}\right) x_{1}+u_{2},
\end{aligned}
$$

where $x=\left(x_{1}, x_{2}\right)$ corresponds to the momentum and the position of the oscillator and $u=\left(u_{1}, u_{2}\right)$ corresponds to the control vector. 
Assuming that the controls are fixed, we may easily find the general solution of the above system. In the case $u_{1}>-1$, the general solution to the system is

$$
\begin{aligned}
& x_{1}=C_{1} \cos \left(\sqrt{1+u_{1}} t\right)+C_{2} \sin \left(\sqrt{1+u_{1}} t\right)+\frac{u_{2}}{1+u_{1}}, \\
& x_{2}=-C_{1} \sqrt{1+u_{1}} \sin \left(\sqrt{1+u_{1}} t\right)+C_{2} \sqrt{1+u_{1}} \cos \left(\sqrt{1+u_{1}} t\right),
\end{aligned}
$$

whereas in the case $u_{1}<-1$, the solution is of the form

$$
\begin{aligned}
& x_{1}=C_{1} \exp \left(\sqrt{\left|1+u_{1}\right|} t\right)+C_{2} \exp \left(-\sqrt{\left|1+u_{1}\right|} t\right)+\frac{u_{2}}{1+u_{1}}, \\
& x_{2}=C_{1}\left|1+u_{1}\right| \exp \left(\sqrt{\left|1+u_{1}\right|} t\right)+C_{2}\left|1+u_{1}\right| \exp \left(-\sqrt{\left|1+u_{1}\right|} t\right) .
\end{aligned}
$$

We now want to solve the problem of choosing the control $u=\left(u_{1}, u_{2}\right)$ in such a way as to minimize the entry time of the solution to a given domain $G$ (surrounding the origin). In order to treat this problem let us recall some definitions from geometric control theory (see, e.g., [3]).

Definition 3.1 (Small time controllability). The system $(f, u)$ is small time controllable in $G$ (STCG) if $G \subseteq \operatorname{int} \mathcal{R}(t), \forall t>0$, where $\mathcal{R}(t)=\left\{x \in \mathbb{R}^{N}: T(x)<t\right\}$. If $G=\{0\}$, we say that the system is small time locally controllable (STLC).

Let us also define the reachable set

$$
\mathcal{R}=\cup_{t>0} \mathcal{R}(t)=\left\{x \in \mathbb{R}^{N}: T(x)<\infty\right\},
$$

that is, the set of points that can reach the target in finite time. As we shall see below, the property of small time controllability is needed for the well-posedness of the formulation of our control problem via the Hamilton-Jacobi equation. There are several criteria to check the STCG of the system. In the present paper we restrict ourselves to the case $N=2$. We have the following

Proposition 3.2. Let $G=\{0\}$. Then the system (3.1) is small time controllable. Furthermore, $T$ is locally Lipschitz continuous in $\mathcal{R}$.

Proof. We use Theorem 1.12 of Chapter IV in 3]. According to that, STCG holds if

$$
\inf _{u \in A} f(0, u) \cdot \gamma<0, \quad \text { for any unit vector } \gamma \text {. }
$$

Indeed, we observe that since $f(0, u)=\left(0, u_{2}\right)$ and $\gamma=(\sin \theta, \cos \theta), f(0, u)=u_{2} \cos \theta$, the infimum of which can be made negative for $u \in A=[-K, K] \times[-K, K]$. Furthermore, according to the same theorem, we have that this condition is necessary and sufficient for the local Lipschitz continuity of $T$ in $\mathcal{R}$.

Such results may be generalized for smooth targets. For instance, the condition

$$
\inf _{u \in A} f(x, u) \cdot n(x)<0, \quad \text { for all } x \in \partial G,
$$

is a sufficient condition for the small time controllability of the system for a smooth target $G$, where $n(x)=\left(n_{1}(x), n_{2}(x)\right)$ is the exterior normal. This condition is rather restrictive. For instance, even for the problem at hand, this condition may fail if we choose $G$ to be a circle encircling the origin. The failure of the condition may be shown in the fact that this inner product can become zero. For example, the above condition for a circular domain with center at the origin does not hold at two isolated points, the points 
of intersection of the circular domain with the $x_{1}$ axis. However, the STCG property for a smooth target may be examined by using more general methods. In particular, for the case of a circular domain, we have the following:

Proposition 3.3. The system (3.1) is STCG in the case of a circular target $G$-centered at the origin.

Proof. If we assume that we have a circular domain with unit radius, then

$$
n(x) \cdot f(x, u)=-u_{1} \sin \theta \cos \theta+u_{2} \sin \theta,
$$

on the boundary $\partial G$. Condition (3.3) is satisfied with the proper choice of controls on any compact subset of $\partial G$ that does not contain the points $x_{2}=0$. Following Proposition 1.2 of [3], we have to control $T(x)$ by a function $\omega_{T}(d(x))$ such that $\lim _{s \rightarrow 0^{+}} \omega_{T}(s)=0$ for all $x$ 's in the neighborhood of the boundary of $\partial G$ in the exterior of $G$, thus obtaining small time controllability. It is enough to establish the above estimate for all points in a neighborhood of the points $( \pm 1,0)$.

Without loss of generality, we consider the points with $x_{2}=0$. Starting from any point outside the $x_{1}$-axis, we can reach the axis in a small time controlled by the distance following the trajectory corresponding to the controls $u_{1}=-1$ and $u_{2}=$ const. Consider a point with $x_{2,0}=0$ and $x_{1,0} \in[1, \delta)$, where $\delta$ is a small positive constant. Setting $u_{1}=-1$ and $u_{2}=$ const, we have

$$
\begin{aligned}
& x_{1}=\frac{1}{2} u_{2} t^{2}+x_{1,0}, \\
& x_{2}=u_{2} t+0 .
\end{aligned}
$$

The trajectory intersects the line $x_{1}=1$ at time $t=\frac{\sqrt{2 d}}{\left|u_{2}\right|}$ with $x_{2}(t)=-\sqrt{2 d}$, where $d=\left|x_{1,0}-1\right|$. We continue the trajectory governed by the controls $u_{2}=0, u_{1}=-1$; then

$$
x_{1}=-\sqrt{2 d} t+1, \quad x_{2}=-\sqrt{2 d} .
$$

The trajectory meets $\partial G$ at the point $(\sqrt{1-2 d},-\sqrt{2 d})$ at time $t=\frac{\sqrt{2 d}}{1+\sqrt{1-2 d}}$.

Small time controllability is not valid for all domains. For example, if $f\left(x_{0}, u\right)$. $n\left(x_{0}\right)>\alpha$, where $x_{0} \in \partial G$ and $\alpha$ is a positive constant, then all trajectories starting from points in a neighborhood of $x_{0}$ in $\partial G$ move away from $G$ so that $T(x)$ cannot meet the zero boundary condition. However, for the needs of the present paper, which aims at controlling the position of the soliton, we can always deform our domain so that our controls can drive the soliton inside $G$. In this direction, we have the following proposition that gives a sufficient condition for small time controllability by choosing the geometry of the domain $G$.

Proposition 3.4. If, for an arbitrary domain $G$, where $n(x)=\left(n_{1}(x), n_{2}(x)\right)$ is the exterior normal with

$$
f(x, u) \cdot n(x)=n_{1} x_{2}-n_{2}\left(1+u_{1}\right) x_{1}+n_{2} u_{2}, \quad x \in \partial G,
$$

we have that

$$
n_{1}\left(x_{1}, x_{2}\right) x_{2}<0, \text { for all } x \in \partial G \text { with } n_{2}\left(x_{1}, x_{2}\right)=0,
$$

then the system (3.1) is STCG for the smooth target $G$. 
Proof. The proof is immediate since we obtain (3.3) by the proper choice of the compact controllability set.

REMARK 3.5. Since we are essentially only interested in controlling the position of the soliton center and not necessarily the velocity of the center, we may deform the domain $G$ as we wish.

We are now ready to formulate the Hamilton-Jacobi equation associated with the minimal time function of our problem. We need the framework of viscosity solutions introduced by Crandall and Lions; see, e.g., 7]. For the sake of completeness, let us repeat the definition of a viscosity solution.

Definition 3.6. We say that a continuous function $T$ solves the Hamilton-Jacobi equation

$$
H(x, T(x), D T(x))=0, \quad x \in G^{c},
$$

in the viscosity sense, where $H$ is a continuous function, if for all $\phi \in C^{1}\left(G^{c}\right)$, we have successively:

- if $(T-\phi)$ has a local maximum at $x_{0} \in G^{c}$, then:

$$
H\left(x_{0}, T\left(x_{0}\right), D \phi\left(x_{0}\right)\right) \leq 0
$$

- if $(T-\phi)$ has a local minimum point at $x_{0} \in G^{c}$, then:

$$
H\left(x_{0}, T\left(x_{0}\right), D \phi\left(x_{0}\right)\right) \geq 0 .
$$

Then we have the following theorem:

THEOREM 3.7. If the target set is closed with compact boundary $\partial G$ and STCG is satisfied, then $T$ is the unique continuous viscosity solution of the Hamilton-Jacobi equation

$$
\sup _{u \in A}\{-f(x, u) \cdot D T\}-1=0, \quad x \in \mathcal{R} \backslash G,
$$

with boundary conditions

$$
\begin{array}{r}
T=0 \quad \text { on } \quad \partial G \\
T(x) \rightarrow+\infty \quad \text { as } \quad x \rightarrow x_{0} \in \partial \mathcal{R}
\end{array}
$$

where $D T=\left(\frac{\partial T}{\partial x_{1}}, \frac{\partial T}{\partial x_{2}}\right)$ and $f(x, u)$ is the vector field corresponding to the system of differential equations.

Proof. For the proof, see Theorem 2.6 of Chapter IV in [3].

Equation (3.8) will be the object of study in this section. Equation (3.8) may be transformed into a Dirichlet boundary value problem using the following transformation. Define the new variable $v(x)$ by

$$
v(x)=\left\{\begin{array}{cc}
1-e^{-T(x)}, & x \in \mathcal{R} \\
1, & x \notin \mathcal{R} .
\end{array}\right.
$$

This satisfies $0 \leq v \leq 1$ for all $x \in \mathbb{R}^{2}$ and once both $T$ and $\mathcal{R}$ are known, the transformation may be inverted to find

$$
T(x)=-\log (1-v(x)), \quad \mathcal{R}=\{x: v(x)<1\} .
$$

In the new variables the Hamilton-Jacobi equation assumes a more suitable form for analysis that can be treated more easily numerically as we will see in the next section. 
We have the following proposition; see [2] (see also Lemma 2.8 and Theorem 2.9 of Chapter IV in [3]):

Proposition 3.8. If the target set is closed with compact boundary $\partial G$ and STCG is satisfied, then $v$ is the unique bounded continuous viscosity solution of the boundary value problem

$$
\begin{array}{rl}
v+\sup _{u \in A}\{-f(x, u) \cdot D v\}-1=0 & x \in G^{c}, \\
v=0 & x \in \partial G .
\end{array}
$$

The solution of this problem characterizes the set of initial conditions from which the system may reach the target as $\mathcal{R}=\{x: v(x)<1\}$. Using the specific form of our control vector field, we have the following:

Proposition 3.9. Under the assumptions of the previous proposition, the function $v$ (Kruzkov transform of the minimal time function $T$ ) for the system at hand is the unique $B C\left(\mathbb{R}^{n}\right)$ viscosity solution of the boundary value problem

$$
\begin{aligned}
v-x_{2} \frac{\partial v}{\partial x_{1}}+x_{1} \frac{\partial v}{\partial x_{2}}+K \sqrt{1+x_{1}^{2}}\left|\frac{\partial v}{\partial x_{2}}\right|-1=0, & x \in G^{c}, \\
v & =0, \quad x \in \partial G .
\end{aligned}
$$

Proof. According to Theorem 2.9, Chapter IV in [3], equation (3.11) holds. We now find the specific form of this equation for the linear oscillator. Assuming bounded controls $\left|u_{i}\right| \leq K, i=1,2$, we may solve the optimization problem $\sup _{u}\{f(x, u) \cdot D v\}$ as follows:

$$
\sup _{u \in A}\{-f(x, u) \cdot D v\}=-x_{2} \frac{\partial v}{\partial x_{1}}+x_{1} \frac{\partial v}{\partial x_{2}}+\sup _{\left|u_{i}\right| \leq K}\{u \cdot F\},
$$

where $u=\left(u_{1}, u_{2}\right)$ and $F=\left(x_{1} \frac{\partial v}{\partial x_{2}},-\frac{\partial v}{\partial x_{2}}\right)$. Since

$$
\sup _{\left|u_{i}\right| \leq K}\{u \cdot F\}=K|F|
$$

and the sup is attained for $u=K \frac{F}{|F|}$, we find that

$$
\sup _{u \in A}\{f(x, u) \cdot D v\}=-x_{2} \frac{\partial v}{\partial x_{1}}+x_{1} \frac{\partial v}{\partial x_{2}}+K \sqrt{1+x_{1}^{2}}\left|\frac{\partial v}{\partial x_{2}}\right| .
$$

Substituting this into equation (3.11) we obtain the desired result.

REMARK 3.10. From the above proof, we see that using the specific form of our Hamiltonian we can compute directly the optimal control corresponding to the position $\left(x_{1}, x_{2}\right)$ via the equation $u=K \frac{F}{|F|}$, that is,

$$
u=\frac{K\left(x_{1} \frac{\partial v}{\partial x_{2}},-\frac{\partial v}{\partial x_{2}}\right)}{\left|\frac{\partial v}{\partial x_{2}}\right| \sqrt{1+x_{1}^{2}}},
$$

as long as the solution of the Hamilton-Jacobi equation $v$ is known as a function of $x=\left(x_{1}, x_{2}\right)$. One could argue that the control is dependent on the position of physical space; the center of the soliton is at time $t$, so that the initial ansatz (2.2) for the control potential which assumes the control $u$ to be purely a function of $t$ is not compatible with the solution of the problem we provide. If this was true, then the controlled equation 
(2.3) upon which we base our analysis would only be valid as an approximation. However, such concerns seem to be unnecessary as the following point of view is more appropriate. The control protocol $u$ given by equation (3.13) is to be considered purely as a function of time as by $x=\left(x_{1}, x_{2}\right)$ in the Hamilton-Jacobi equation we denote the initial condition of the controlled system (in our case the initial position of the soliton center). Therefore, after finding the solution of the Hamilton-Jacobi equation, the calculation of the optimal control performed via equation (3.13) gives $u$ as a function of $t$, that is, $u=u\left(t ; x_{0}\right)$ where $x_{0}$ denotes the initial position of the soliton center. The control is thus not a feedback type of control where $u$ depends on the instantaneous position of the soliton center $x$ and which would be incompatible with our initial ansatz for the control potential. To make the situation more clear, on the physical problem we wish to study, we consider the problem we solve corresponds to the case where the control engineer observes the initial position of the soliton center $x_{0}$ and then computes the control protocol $u=u\left(t ; x_{0}\right)$, which is then applied unchanged until the soliton reaches the chosen target. This is not what would happen in the case of the feedback control where the control engineer would adjust the potential using a different protocol $u^{\prime}=u(x(t))$ depending on the instantaneous position of the soliton center at time $t, x(t)$.

REMARK 3.11. The Hamilton-Jacobi equation for the Kruzkov transform of the minimum time function may be written in terms of polar coordinates in simpler form as

$$
v+\frac{\partial v}{\partial \theta}+K \sqrt{1+r^{2} \cos ^{2} \theta}\left|\frac{\partial v}{\partial r} \sin \theta+\frac{\partial v}{\partial \theta} \cos \theta\right|-1=0,
$$

where we look for solutions that are periodic in the variable $\theta$. The above form of the equation may also be useful for asymptotic analysis.

The STCG assumption is very important. If it is not satisfied, then we do not expect in general to have a continuous minimal time function $T(x)$. The zero boundary condition has to be satisfied in the viscosity sense and we need the theory of discontinuous viscosity solutions leading to uniqueness problems. In this case for example, the first arrival time $\hat{T}(x)$ in the interior of $G$ is also a weak solution of our problem; see, e.g., Chapter $\mathrm{V}$ in [3]. However, even in this case we have a "uniqueness" result stating that all viscosity solutions have the same lower semicontinuous envelope (see [5] ).

On the other hand, strong uniqueness is required for the numerical investigation of (3.12) and for the purposes of the present paper we can choose our target domain $G$ according to Propositions 3.4-3.5 so as to guarantee STCG. In the next section we study numerically (3.12) by using a proper monotone scheme and assuming that STCG holds.

\section{A numerical scheme for the solution of the Hamilton-Jacobi equation.}

In this section we implement a numerical scheme for the calculation of the Kruzkov transform of the minimal time $v$ and the minimal time itself. Naturally, we have to restrict the problem to a bounded set $\Omega$ containing the target set and solve (3.12) in $\Omega \backslash G$. Our scheme is motivated by the finite difference schemes used in the approximation of solutions of Hamilton-Jacobi type, e.g., 8]. We let $\Omega=(-L, L)^{2}$ denote a square domain of side $2 L>0$ containing the target set $G$. Given the mesh sizes $\Delta x_{1}, \Delta x_{2}$, we denote by $V_{i j}$ the numerical approximation of the solution of (3.12) at the point 
$\left(x_{1, i}, x_{2, j}\right)=\left(-L+i \Delta x_{1},-L+j \Delta x_{2}\right), i=0, \ldots, I, j=0, \ldots, J$, with $I=2 L / \Delta x_{1}$, $J=2 L / \Delta x_{2}$. Moreover, we define the index sets

$$
\begin{aligned}
Q & =\left\{(i, j):\left(x_{1, i}, x_{2, j}\right) \in \Omega \backslash G\right\}, \\
\partial Q & =\left\{(i, j):\left(x_{1, i}, x_{2, j}\right) \in \partial \Omega\right\}, \\
Q_{G} & =\left\{(i, j):\left(x_{1, i}, x_{2, j}\right) \in G\right\},
\end{aligned}
$$

and the standard difference operators

$$
\begin{aligned}
\Delta_{x_{1}}^{+} U_{i j} & =\frac{U_{i+1 j}-U_{i j}}{\Delta x_{1}}, & \Delta_{x_{1}}^{-} U_{i j} & =\frac{U_{i j}-U_{i-1 j}}{\Delta x_{1}}, \\
\Delta_{x_{2}}^{+} U_{i j} & =\frac{U_{i j+1}-U_{i j}}{\Delta x_{2}}, & \Delta_{x_{2}}^{-} U_{i j} & =\frac{U_{i j}-U_{i j-1}}{\Delta x_{2}},
\end{aligned}
$$

for any approximation $U$ and $(i, j) \in Q$. Finally, for any $(i, j) \in Q$, we define $g_{i j}(x, w, a, b, c, d)$ by

$$
\begin{gathered}
g_{i j}(x, w, a, b, c, d)=w \\
-\left[x_{2, j}\right]^{+} a+\left[x_{2, j}\right]^{-} b-\left[x_{1, i}\right]^{-} c+\left[x_{1, i}\right]^{+} d \\
+K \sqrt{1+x_{1, i}^{2}} \max \left\{c^{+}, d^{-}\right\}-1,
\end{gathered}
$$

where $[x]^{+}$and $[x]^{-}$are the positive and negative parts of $x$, respectively. We require that the numerical approximation $V$ of (3.12) satisfy

$$
\begin{gathered}
g_{i j}\left(x, V_{i j}, \Delta_{x_{1}}^{-} V_{i j}, \Delta_{x_{1}}^{+} V_{i j}, \Delta_{x_{2}}^{-} V_{i j}, \Delta_{x_{2}}^{+} V_{i j}\right)=0, \quad \forall(i, j) \in Q, \\
V_{i j}=1, \quad \forall(i, j) \in \partial Q, \quad V_{i j}=0, \quad \forall(i, j) \in Q_{G} .
\end{gathered}
$$

To prove the existence of a solution of (4.1)-(4.2), we shall use the results of Barles and Souganidis 6].

Proposition 4.1. Under the assumptions of Propositions 3.4 and 3.5, the numerical scheme defined by (4.1)-(4.2) converges to the unique viscosity solution of (3.12).

Proof. Consistency is immediate from the definition of the scheme. For the monotonicity, we observe that for all $\left(x_{1}, x_{2}\right) \in \Omega$ and all grid functions $U, V$ such that $U \geq V$ with $V_{i j}=U_{i j}=w$, we have

$$
\begin{aligned}
& g_{i j}\left(x, U, \frac{w-U_{i-1 j}}{\Delta x_{1}}, \frac{U_{i+1 j}-w}{\Delta x_{1}}, \frac{w-U_{i j-1}}{\Delta x_{2}}, \frac{U_{i j+1}-w}{\Delta x_{2}}\right) \\
& \quad \leq g_{i j}\left(x, V, \frac{w-V_{i-1 j}}{\Delta x_{1}}, \frac{V_{i+1 j}-w}{\Delta x_{1}}, \frac{w-V_{i j-1}}{\Delta x_{2}}, \frac{V_{i j+1}-w}{\Delta x_{2}}\right),
\end{aligned}
$$


so that the numerical scheme is monotone in the sense of [6]. The scheme is stable in the sense that for all $\Delta x_{1}, \Delta x_{2}>0$, a discrete solution $V$ exists (see below) and is bounded independently of $\Delta x_{1}, \Delta x_{2}$. The existence of an upper bound is obvious from the monotonicity of the scheme while a lower bound is obtained as follows: if $V$ has a strong negative minimum at $\left(x_{1, i}, x_{2, j}\right)$, then, solving (4.1) for $w=V_{i j}$, we obtain, e.g., in the case $x_{1, i}>0, x_{2, j}>0$,

$$
V_{i j}=\left|x_{2, j}\right| \frac{V_{i j+1}-V_{i j}}{\Delta x_{1}}-\left|x_{1, i}\right| \frac{V_{i j}-V_{i j-1}}{\Delta x_{2}}+1,
$$

which contradicts the sign of the right-hand side of the above equation. The other cases are handled similarly.

Next we present an algorithm for computing the solution of (4.1)-(4.2). We note first that if $V$ is given at each point $(k, l) \neq(i, j)$, it is possible to find $w \in \mathbb{R}$ such that $V_{i j}=w$ and $g_{i j}(x, V, a, b, c, d)=0$. In fact, with

$$
m=\min \left\{V_{i j-1}, V_{i j+1}\right\}
$$

and

$$
\begin{gathered}
L=1+\frac{\left|x_{1}\right|}{\Delta x_{2}}+\frac{\left|x_{2}\right|}{\Delta x_{1}}, \\
R=1+\frac{\left[x_{1}\right]^{+} V_{i, j-1}+\left[x_{1}\right]^{-} V_{i, j+1}}{\Delta x_{2}}+\frac{\left[x_{2}\right]^{+} V_{i+1, j}+\left[x_{2}\right]^{-} V_{i-1, j}}{\Delta x_{1}},
\end{gathered}
$$

it is readily checked that

$$
V_{i j}= \begin{cases}R / L, & \text { if } m L \geq R, \\ \left(R+\frac{m K \sqrt{1+x_{1}^{2}}}{\Delta x_{2}}\right) /\left(L+\frac{K \sqrt{1+x_{1}^{2}}}{\Delta x_{2}}\right), & \text { otherwise. }\end{cases}
$$

Our algorithm proceeds as follows: Given an initial approximation $U_{i j}^{0},(i, j) \in Q$ such that $g_{i j}\left(x, U^{0}, a, b, c, d\right) \leq 0$ and $n \geq 0$, we compute a new approximation $U^{n+1}$ by $U_{i j}^{n+1}=V_{i j},(i, j) \in Q$, where $V_{i j}$ is computed from (4.3).

In the numerical experiments reported here we imposed a regular grid of size $\Delta x_{1}=$ $\Delta x_{2}=0.0125$ on the square $[-2,2]^{2}$ and solved the boundary problem (3.12) using the scheme (4.1)-(4.2). We used the criterion $\left|V_{i j}^{n+1}-V_{i j}^{n}\right|<10^{-5}$ to control the number of iterations needed for the update of each grid point. We found out that only a small number of iterations were necessary in order to satisfy this criterion, in all cases between four and six. We experimented with the following target sets:

$$
\begin{gathered}
G_{c}=\left\{(x, y): \frac{13}{2} x^{2}+\frac{13}{2} y^{2}<1\right\}, \\
G_{ \pm}=\left\{(x, y): \frac{13}{2} x^{2} \pm 5 x y+\frac{13}{2} y^{2}<1\right\} .
\end{gathered}
$$


$\partial G_{c}$ is a circle centered at the origin and with radius $\sqrt{2 / 13} . \partial G_{ \pm}$is the ellipse $4 \xi^{2}+$ $9 \eta^{2}=36$ rotated by an angle of $\pm 45^{\circ}$. In Figures 13 we plotted the minimal time function corresponding to each target set along with the contour levels corresponding to times $0.1,0.2,0.3$, and 0.4 .

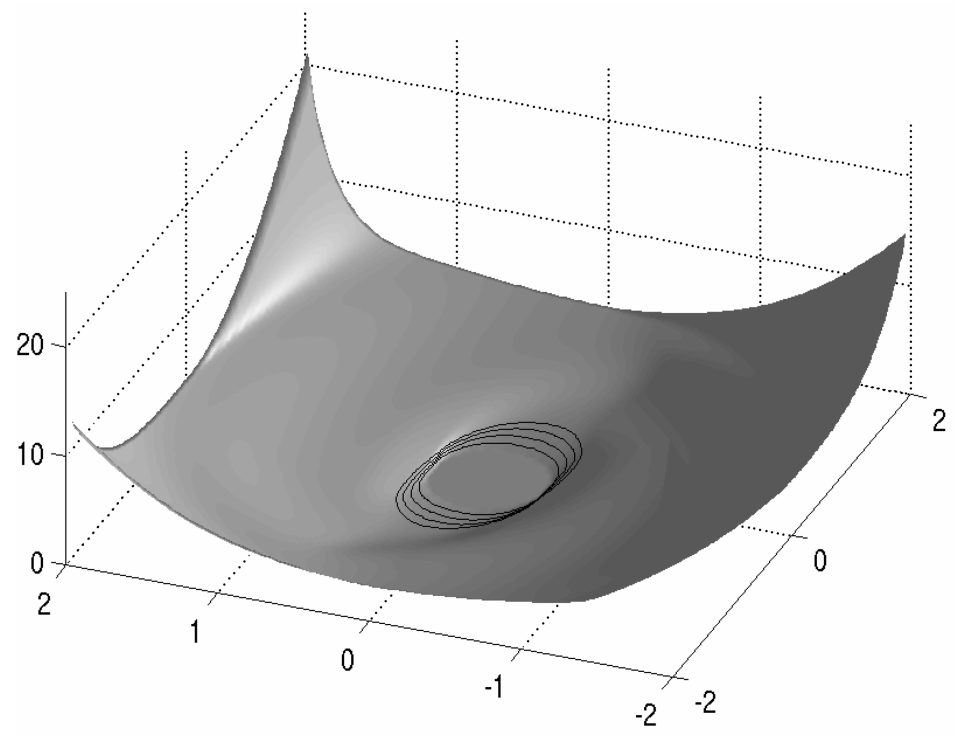

FIG. 1. Surface plot of the minimal time for the target set $G_{c}$

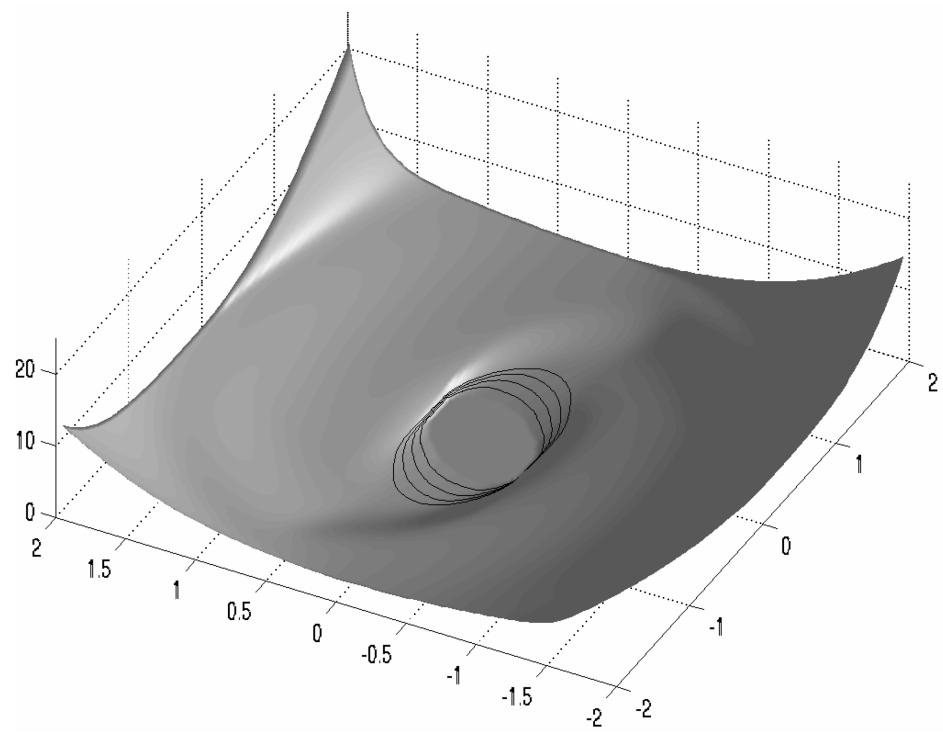

FIG. 2. Computed minimal time for the target set $G_{-}$ 


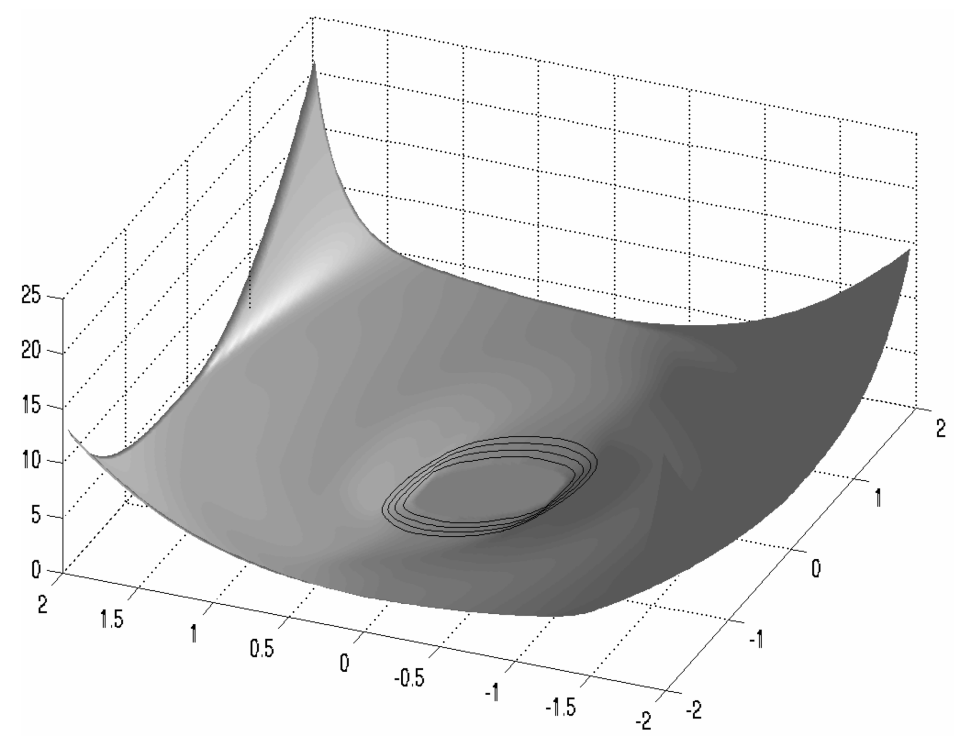

FIG. 3. Computed minimal time for the target set $G_{+}$

The numerical results presented in Figure 1 represent the optimal time in the case of the circular target set $G_{c}$. Due to small time local controllability given in Proposition 3.2, the numerical scheme 4.1 -4.2 converges to the unique, continuous viscosity solution of (3.12). In Figures 2 and 3 the target sets are the ellipses $G_{ \pm}$. In Figure 2 we depict the numerical results in the case of the ellipse $G_{-}$, which is the one rotated by an angle of $-45^{\circ}$ for which conditions of Proposition 3.3 apply, thus obtaining a continuous viscosity solution. So, $G_{-}$is a proper target set into which we can drive our soliton. In contrast to that, small time controllability is violated in the case of the ellipse $G_{+}$. At the points $x \in \partial G_{+}$for which $n_{2}\left(x_{1}, x_{2}\right)=0$, we have $n_{1}\left(x_{1}, x_{2}\right) x_{2} \geq 0$, so that all trajectories starting from neighbouring points drive the system away from the boundary and the optimal time is not zero. The zero boundary condition is violated, leading to a discontinuous viscosity solution and giving rise to a uniqueness question. Such discontinuity is depicted in Figure 3, where we clearly notice that our numerical scheme (4.1) - (4.2) is following the discontinuous solution and level curves are compressed in the discontinuous region. A better depiction of the discontinuity is given in Tables 1-2, where we show values of the solution on grid points around the points of discontinuity that clearly exhibit an abrupt change in their values. The smoothing effect appearing in these tables is due to the diffusivity introduced by our numerical scheme.

The following numerical results give the minimum time needed to drive the soliton into the target set $G$ as a function of initial position $\left(x_{1}, x_{2}\right)$. We next address the important question of computing the approximate optimal control for the trapping of the soliton, which is of particular interest in the applications. Using the specific form of our Hamiltonian, we can compute directly the optimal control corresponding to the 


\begin{tabular}{c||c|c|c|c|c}
\hline$y / x$ & 0.400 & 0.425 & 0.450 & 0.475 & 0.500 \\
\hline \hline 0.075 & 0.000 & 0.382 & 0.478 & 0.538 & 0.583 \\
\hline 0.100 & 0.000 & 0.398 & 0.490 & 0.548 & 0.592 \\
\hline 0.125 & 0.000 & 0.415 & 0.502 & 0.559 & 0.600 \\
\hline 0.150 & 0.000 & 0.433 & 0.515 & 0.569 & 0.609 \\
\hline 0.175 & 0.000 & 0.451 & 0.529 & 0.580 & 0.618 \\
\hline 0.200 & 0.000 & 0.469 & 0.542 & 0.591 & 0.628 \\
\hline 0.225 & 0.000 & 0.488 & 0.556 & 0.602 & 0.637 \\
\hline 0.250 & 0.000 & 0.507 & 0.570 & 0.614 & 0.646 \\
\hline
\end{tabular}

TABLE 1. Computed minimal time in a neighborhood of the point $(0.424918,0.163401)$ on the ellipse $G_{+}$.

\begin{tabular}{c||c|c|c|c|c}
\hline$y / x$ & 0.400 & 0.425 & 0.450 & 0.475 & 0.500 \\
\hline \hline-0.250 & 0.000 & 0.090 & 0.167 & 0.235 & 0.295 \\
\hline-0.225 & 0.000 & 0.091 & 0.169 & 0.237 & 0.298 \\
\hline-0.200 & 0.000 & 0.094 & 0.173 & 0.241 & 0.301 \\
\hline-0.175 & 0.000 & 0.097 & 0.177 & 0.245 & 0.306 \\
\hline-0.150 & 0.000 & 0.102 & 0.182 & 0.251 & 0.311 \\
\hline-0.125 & 0.000 & 0.108 & 0.189 & 0.257 & 0.317 \\
\hline-0.100 & 0.000 & 0.115 & 0.197 & 0.264 & 0.324 \\
\hline-0.075 & 0.000 & 0.123 & 0.205 & 0.273 & 0.331 \\
\hline
\end{tabular}

TABLE 2. Computed minimal time in a neighborhood of the point $(0.424918,-0.163401)$ on the ellipse $G_{-}$.

position $\left(x_{1}, x_{2}\right)$ via the equation $u=K \frac{F}{|F|}$; see (3.13). Using the numerical scheme (4.1)(4.2), it is possible to compute the optimal control $v$ using appropriate finite differencetype approximations of the derivatives appearing in (3.13). This, along with detailed experiments involving higher order approximations of (3.12), will be the subject of future investigation.

5. Conclusions. In this paper we have presented a method for the solution of the soliton control problem. In particular, we studied the problem of driving a soliton within a chosen target $G$ in the minimum time by applying a properly selected external potential which acts as the control. The problem was approached by the reduction of the equation of motion for the soliton center to that of a controlled oscillator, which was then treated using viscosity solutions of the appropriate Hamilton-Jacobi equation. A numerical scheme is proposed which allows calculation of the minimum time. An expression for the control protocol, in terms of the Kruzkov transform of the minimal time, is also given, which is feasible to treat numerically but would require more elaborate schemes than the minimal time. 
From the physical point of view, the calculation of the minimum time needed in order to drive the soliton into the desired target set $G$ is by itself an interesting quantity for the purposes of mathematical modeling of soliton systems, as it provides us with a lower bound for the time needed in order for the system to reach the desired state. The information of how this quantity depends on the various parameters characterizing the system (e.g., dispersion coefficient, etc.) is very useful for the design experiments and devices in fields such as fiber optics or Bose-Einstein condensation.

Our ODE approach can be extended for the study of the higher moments of the soliton apart from the first (i.e., the soliton center) but approximations will have to be introduced. It can also be extended to include the presence of noise. This will lead to second order Hamilton-Jacobi type equations and appropriate modifications in the definition of viscosity solutions and the numerical schemes are needed. This problem is under active consideration and we hope to report on it in the near future.

Acknowledgements. The authors gratefully acknowledge the support of the European Union research networks Hyperbolic and Kinetic Equations under contract number HPRN-CT-2002-00282 and that of Fronts and Singularities under contract number HPRN-CT-2002-00274. The first author thanks Prof. M. Bardi for a fruitful discussion concerning target problems in control theory.

\section{REFERENCES}

[1] F. K. Abdullaev, J. C. Bronski and G. Papanicolaou, Soliton perturbations and the random Kepler problem, Physica D 135 (2000), 369-386. MR1731507 (2000j:35237)

[2] M. Bardi, A boundary value problem for the minimum time function, SIAM J. Control and Optimization 27 (1989), 776-785. MR1001919|(90i:49034)

[3] M. Bardi and I. Capuzzo-Dolcetta, Optimal Control and Viscosity Solutions of Hamilton-JacobiBellman Equations, Birkhäuser Boston, Inc., Boston, 1997. MR.1484411 (99e:49001)

[4] M. Bardi and M. Falcone, An approximation scheme for the minimum time function, SIAM J. Control and Optimization 28 (1990), 950-965. MR.1051632(91d:49029)

[5] G. Barles and B. Perthame, Comparison Principle for Dirichlet-type Hamilton-Jacobi Equations and Singular Perturbations of Degenerate Elliptic Equations, Appl. Math. Optim. 21 (1990), 21-44. MR.1014943 (90m:35017)

[6] G. Barles and P. E. Souganidis, Convergence of approximation schemes for fully nonlinear second order equations, Asymptotic Analysis 4 (1991), 271-283. MR.1115933 (92d:35137)

[7] M. G. Crandall, H. Ishii, and P.-L. Lions, User's guide to viscosity solutions of second order partial differential equations, Bull. Amer. Math. Soc. 27 (1992), 1-67. MR1118699 (92j:35050)

[8] M. G. Crandall and P.-L. Lions, Two approximations of solutions of Hamilton-Jacobi equations, Math. Comp. 43 (1984), 1-19. MR0744921|(86j:65121)

[9] J. Garnier, Asymptotic transmission of solitons through random media, SIAM J. Appl. Math. 58 (1998), 1969-1995. MR1644311 (99d:35151)

[10] A. Hasegawa, Y. Kodama and Y. Kodama, Solitons in optical communications, Oxford Series in Optical and Imaging Science 7, Clarendon Press, Oxford 1997.

[11] L. Pitaevskii and S. Stringeri, Bose-Einstein condensation, International Series of Monographs on Physics, Clarendon Press, Oxford 2003. MR2012737 (2004i:82038)

[12] E. Rouy and A. Tourin, A viscosity solution approach to shape-from-shading, SIAM J. Num. Anal. 29 (1992), 867-884. MR1163361 (93d:65019)

[13] A. N. Yannacopoulos, D. J. Frantzeskakis, C. Polymilis and K. Hizanidis, Conditions for soliton trapping in random potentials using Lyapunov exponents of stochastic ODEs, Phys. Lett. A 271 (2000), 334-340. MR1773301 (2001c:82039) 
[14] A. N. Yannacopoulos, D. J. Frantzeskakis, C. Polymilis and K. Hizanidis, Motion of 2D Schrödinger Solitary Waves in the Presence of Random External Potentials, Physica Scr. 65 (2002), 363-368.

[15] H. Zhao, A fast sweeping method for eikonal equations, to appear in Math. Comp. 